\title{
Analysis of Wind Turbines Blockage on Doppler Weather Radar Beams
}

\author{
Aniceto Belmonte and Xavier Fabregas
}

\begin{abstract}
The split-step solution to the parabolic wave equation describing beam propagation permits examination of the signal degradation in a Doppler weather radar caused by wind turbines blockage under general atmospheric conditions and at arbitrary transmitter and receiver configurations. At radar wavelengths, an understanding of turbine and terrain obscuration effects is essential for deciding the reliability of radar measurements affected by blockage.
\end{abstract}

Index Terms-Beam propagation methods, Doppler radar, splitstep solution, wind turbines.

\section{INTRODUCTION}

$\mathbf{E}$ VEN partial blockage of meteorological Doppler radar beams by any natural or man-made obstacle results in a deterioration of the radar performance and loss of sensitivity to atmospheric precipitation and wind conditions. In this respect, due to its high-level radar cross sections and the rotation of its blades, wind turbines have a potentially strong impact on Doppler radar capacities. In many locations, the growing number of wind turbines close to those geographical elevated spots until now only occupied by weather radars is becoming a serious threat to the reliability of radar meteorological measurements.

Wind turbines can impact coherent Doppler radars if they are within the radar's line of sight [1]. Within a few kilometers from the radar, they are close enough to partially block a significant percentage of the beam and attenuate signal downrange of the wind turbine. They can also reflect energy back to radar and appear as clutter on the radar image and contaminate the base reflectivity data [2], [3]. Finally, if the turbine blades are moving, they can impact the velocity and spectrum width data [4]. In this study, we focus our efforts in the analysis and quantification of blockage effects.

To analyze the effects of wind turbines blockage on coherent weather radar performance in a realistic way, it is necessary to

\footnotetext{
Manuscript received May 27, 2010; accepted June 30, 2010. Date of publication July 15, 2010; date of current version July 26,2010 . This work was supported in part by the Catalonia Department for Environment, Meteorological Service Technical Contract 01143-2008 and the Spanish Department of Science and Innovation MICINN grants TEC 2009-10025 and TEC 2008-06764C02-01.

The authors are with the Department of Signal Theory and Communications, Technical University of Catalonia, 08034 Barcelona, Spain (e-mail: belmonte@tsc.upc.edu).

Color versions of one or more of the figures in this letter are available online at http://ieeexplore.ieee.org.

Digital Object Identifier 10.1109/LAWP.2010.2057238
}

consider the use of simulations of beam propagation in three-dimensional media. This study shows the viability of the splitstep solution to simulate the propagation phenomena while respecting those well-established numerical requirements for a simulation of given accuracy [5]. Though the analysis shown in this letter is indebted to study blockage effects, the approach and conclusions of the propagation method are applicable to other areas dealing with wind turbines impact on coherent weather radars, i.e., clutter and spectrum effects. The authors anticipate addressing them in a following study.

\section{Propagation And Analysis Technique}

The parabolic approximation to the wave equation and its split-step solution [5] has been used extensively in a variety of context to simulate propagation phenomena in both deterministic and random media (e.g., [6]-[8]). The split-step method offers a numerically efficient, full-wave solution to the field because of the implementation of discrete Fourier algorithms in a computer model. While computer execution times increase for increasing frequency and large propagation distances, with the availability of more powerful computing resources, such simulations have been extended to a variety of problems, and there is an extensive body of applications of this technique to engineering problems. To the best of our knowledge, our analysis uses for the first time the split-step approach to the parabolic equation to consider the turbine blockage on weather radar performance.

Physically, the split-step technique corresponds to dividing the medium into slabs, each of which introduces a spatially varying contribution to the phase defined by the atmospheric refraction in the slab, and the radar wavebeam is then propagated through a uniform medium from screen to screen. Although wavefront bends and radar beams are normally refracted downward toward the Earth, a curvature transformation is made that effectively maps the range-dependent terrain coordinate system to a flat or smooth earth coordinate system.

While plane waves are often a useful approximation in many circumstances, in this analysis a more point-like source with significant angular spread is required. This effect may be modeled by propagating in spherical coordinates. This approach, where the propagation medium is divided into spherical shells, keeps angular resolution constant. It allows us high resolution near the source and decreasing resolution with increasing sampling interval as the propagation distance increases.

The electric field envelope $U(\boldsymbol{r}, R)$ characterizes the radar beam considered in this study, which is assumed to be varying relatively slowly in range $R$. If $U_{n}$ is the solution at range $R_{n}$, by using the split-step technique to the parabolic wave equation, 
the solution $U_{n+1}$ at range $R_{n}+\Delta R$ can be approximated analytically in terms of $U_{n}$ with a second-order accuracy by the symmetric split operator

$$
\begin{aligned}
U_{n+1}= & \exp \left(-\frac{j}{2 k} \frac{\Delta R}{2} \nabla_{\perp}^{2}\right) \exp (-j k N) \\
& \times \exp \left(-\frac{j}{2 k} \frac{\Delta R}{2} \nabla_{\perp}^{2}\right) U_{n}
\end{aligned}
$$

where $\Delta_{\perp}$ is the transverse part of the Laplacian operator. This solution allows us to apply a numerical scheme in which the field has to be propagated twice a distance $\Delta R / 2$ in free space by using the operator

$$
S_{\mathrm{F}}=\exp \left(-\frac{j}{2 k} \frac{\Delta R}{2} \nabla_{\perp}^{2}\right)
$$

and the medium has been reduced to a series of independent phase screens defined by

$$
S_{\mathrm{P}}=\exp (-j k N) .
$$

Here, $N$ reflects effect of the medium refractive index. When the radar beam encounters an obstacle, i.e., terrain and wind turbines, power is removed from the beam and a radio shadow appears behind the obstacle. The effect is readily simulated by defining a numerical mask that, collocated with the medium phase screen, properly blocks the propagation field $U_{n}$ at the right transversal coordinates $\boldsymbol{r}$. This occultation effect builds up by diffraction over many consequent slabs and produces wave field distortions.

The three operators defining solution (1) are applied sequentially so that it can be solved separately. The free-space propagation operator (2) can be expressed in Fourier transform space as a simple phase change

$$
\begin{aligned}
& u\left(\boldsymbol{K}, R_{n}+\frac{\Delta R}{2}\right) \\
& \quad=\exp \left[j k\left(\frac{1}{2 R_{n}}-\frac{1}{2 R_{n}+\Delta R}\right)|\boldsymbol{K}|^{2}\right] u\left(\boldsymbol{K}, R_{n}\right)
\end{aligned}
$$

where $u(K, R)$ is the Fourier transform of the electric field envelope $U(\boldsymbol{r}, R)$. Using the fast Fourier transform (FFT) allows numerical propagation of the field from screen to screen in a very efficient way.

The most important limitations on the applicability of the simulations are a consequence of using discrete FFT algorithms for solving numerically the free-space propagation (4). Because of the periodicity of the fields and the fact that the numerical grid must be of finite extension, aliasing and windowing may produce serious errors on the outputs of the simulations [5], [8]. The problem of choosing the appropriate transversal grid sampling interval $\Delta$ and grid extension $M \Delta \times M \Delta$ is addressed by using classical signal processing methods. We utilize a square grid with identical sampling $\Delta$ for both transversal axis, and the number of points $M$ is a power of two for assuring the efficiency of the discrete Fourier transform computation using a FFT based on the classical Danielson-Lanczos lemma. The Winograd transform algorithm could help to relax this condition, allowing $M$ not being a power of two slightly degrading in speed.
If the radiation field distribution is to be calculated, all its spatial scales must be properly sampled. Likewise, the grid has to be large enough to allow the diffraction-broadened radar beam. We have considered these two conditions and used a numerical transversal grid of $256 \times 256$ points with angular resolution ranging from 20 arcseconds to 40 arcseconds (occasionally a grid of $512 \times 512$ points with angular resolution ranging from 10 to 30 arcseconds was considered). We have also simulated the continuous propagation medium with a high longitudinal sampling that uses a two-dimensional phase screen every $100 \mathrm{~m}$. Subject to the limitations of the simulation technique, we considered radar paths of different ranges (from 1 to $20 \mathrm{~km}$ ).

Fields are numerically obtained at every propagation step, allowing detailed investigation of amplitude and phase distortions along the path. The radiation pattern of the radar antenna reveals energy distribution into complex lobe structures. Now, the energy radiated by the radar antenna is not mainly concentrated around the axis of the nominal main lobe. It is of engineering interest to be able to predict how these distorted radiation patterns affects to weather radar returns.

\section{WIND TURBINE BLOCKAGE}

In this analysis, we have considered typical modern horizontal-axis, three-bladed wind turbines used in wind farms for commercial production of electric power. The main rotor shaft and electrical generator is at the top of a tubular steel tower $80 \mathrm{~m}$ tall with a section expanding from $2 \mathrm{~m}$ at the top to $4 \mathrm{~m}$ at the base. Each of the blades connected to the shaft is $45-\mathrm{m}$ long and 2 -m broad. In our numerical analysis, we also include the effect of a supposedly flat, uniform terrain at the bottom of the turbine tower. As we intend to focus on wind turbine blockage alone, no other effects from terrain met by the radar beam before or after the turbine site are contemplated in the results presented.

In our estimations, and without loss of generality, we consider conventional C-band Doppler weather radars with $5.6 \mathrm{GHz}$ operating frequency, $45 \mathrm{~dB}$ antenna gain, and $1.2^{\circ}$ half-power beamwidth. In most cases, neglecting sidelobes, we can assume that the power distribution in the main lobe can be properly characterized by a Gaussian beam. Other radar characteristics, such as pulsewidth, peak transmitted power, or receiver sensitivity, are not relevant to this blockage analysis. C-band weather radars send high-directional pulses of microwave radiation that spread out as they move away from the radar. At close ranges, radar pulses are just a few meters across and, consequently, they are prone to be blocked by obstacles in the proximity of the radar antennas. At far distances, the decreasing spatial resolution of the beams makes them less sensitive to blockage.

Figs. 1 and 2 show the effects of wind turbines blockage on characteristic C-band Doppler weather radars. In these plots, we assume the slightly worst-case scenario where the three turbine blades are visible to the radar. From Fig. 1, it is clear that wind turbines within a few kilometers of a meteorological radar can prevent the radar's beam from properly forming, thus causing significant radar estimation errors downrange from the turbines. In this estimation, terrain and wind turbine occultation effects in the $\mathrm{C}$-band weather radar antenna radiation pattern are shown when the wind turbine was positioned $3 \mathrm{~km}$ away from the radar (upper plot) and the complex radiation structure (middle plot) 

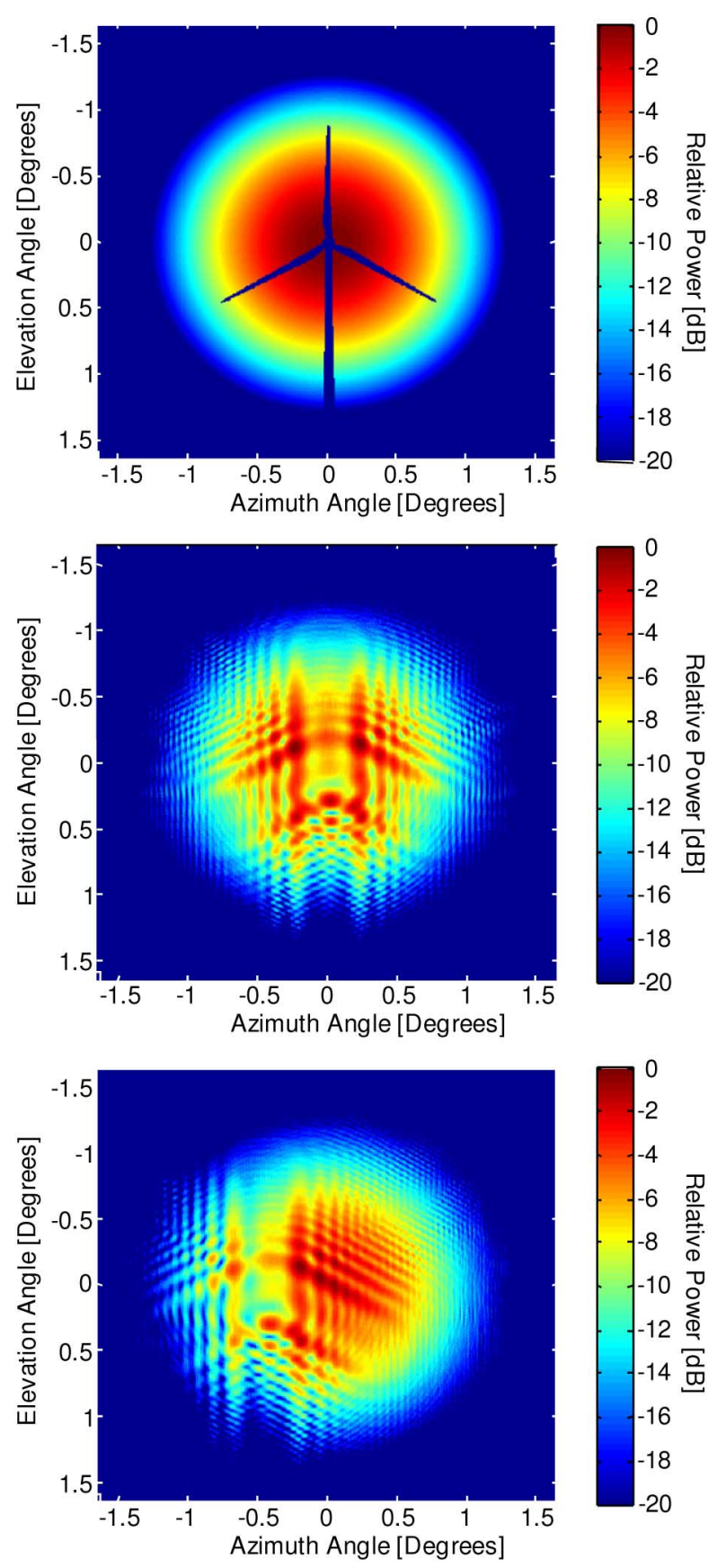

Fig. 1. Terrain and wind turbine occultation effects in a C-band weather radar antenna radiation pattern. We show the undisturbed intensity beam distribution (upper plot) before hitting on-axis the wind turbine considered in the analysis. In these simulations, the wind turbine was positioned $3 \mathrm{~km}$ away from the radar, and the complex radiation structure (middle plot) was estimated $10 \mathrm{~km}$ downrange from the turbine. As expected, this radiation pattern changes considerably when the turbine is hit off-axis by the radar beam (lower plot).

was estimated $10 \mathrm{~km}$ downrange from the turbine. As expected, this radiation pattern change considerably when the turbine is hit off-axis by the radar beam (lower plot). As shown in Fig. 1, the number of spurious, secondary radiation lobes in the turbine-modified antenna radiation pattern is very large, in particular at short distances, and remains sufficiently significant even at large ranges down from the turbine. It may call into question
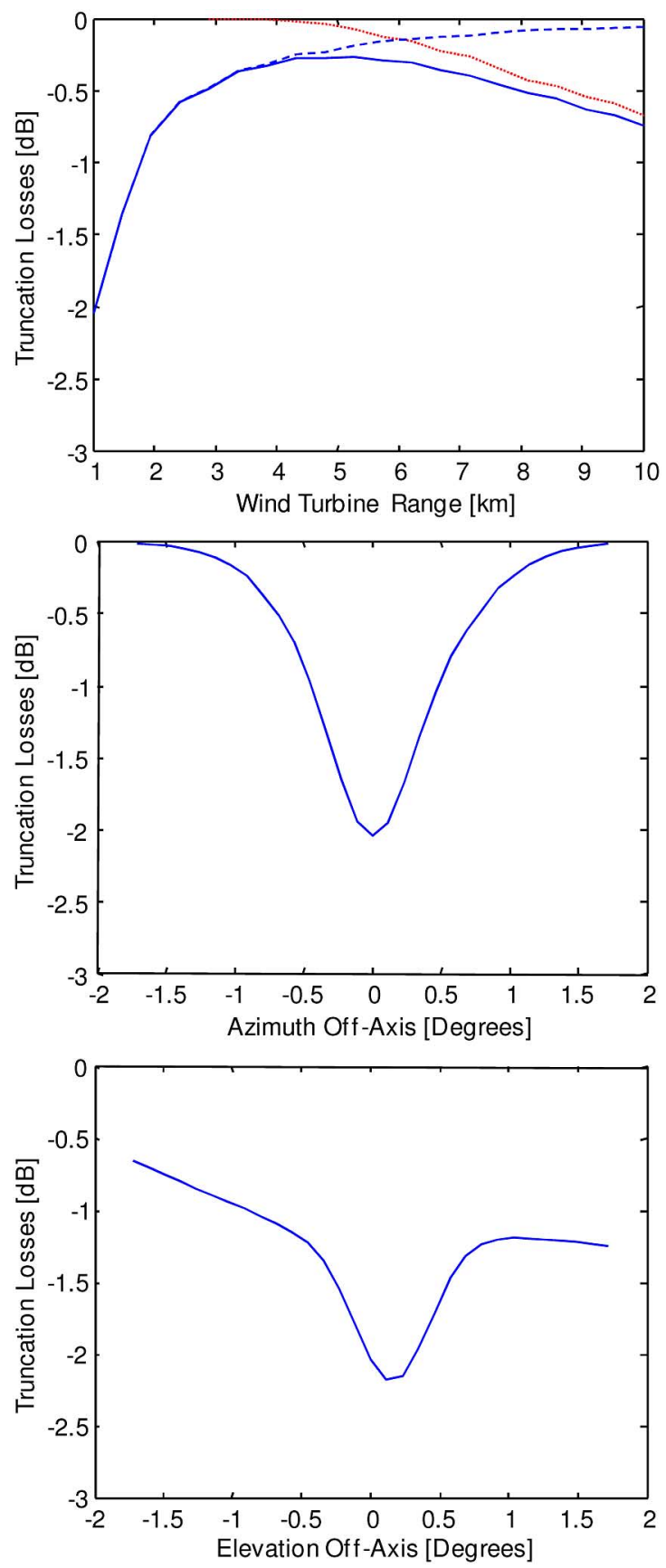

Fig. 2. Terrain and wind turbine occultation effects in a C-band weather radar. In the upper plot, terrain plus turbine truncation losses, in decibels, are shown as a function of the separation between radar and turbine (blue line). We assume that the turbine is hit on-axis by the radar beam (see upper plot in Fig. 1). Terrain truncation losses (dotted line) and wind turbine occultation losses (dashed line) are also shown separately. In the middle plot, for a 1-km-away wind turbine, we plot occultation losses as a function of the azimuth off-axis beam impeding angle over the turbine. In the lower plot, for the same 1-km-away wind turbine, we also plot occultation losses as a function of the elevation off-axis beam impeding angle.

the whole reliability of these radar measurements. In some applications, when low-level signals in clear air conditions need to be measured, the radar system may be extremely affected by areas where the Doppler signal is distorted by the presence of nearby wind turbines. 
In Fig. 2, occultation losses due to terrain and wind turbines are shown. Occultation may become an important attenuation contribution downrange of the wind turbine. In the upper plot, terrain and wind turbine truncation losses, in decibels, are shown as a function of the separation between radar and turbine (solid line). We assume that the turbine is hit on-axis by the radar beam, as shown in the upper plot of Fig. 1. Terrain truncation losses (dotted line) and wind turbine occultation losses (dashed line) are also shown separately. In the middle plot, for a $1-\mathrm{km}$ away wind turbine, we plot occultation losses as a function of the azimuth off-axis beam impeding angle over the turbine. In the lower plot, for the same 1-km-away wind turbine, we also plot occultation losses as a function of the elevation off-axis beam impeding angle.

It thus appears that, in the case of the wind turbines analyzed in this study, the impact on the radar signal will be relevant at all azimuths and elevations for a turbine located up to $3 \mathrm{~km}$ away (Fig. 2, upper plot). As much as 2-dB truncation losses may be observed in any real Doppler radar working under such field-of-view restrictions. For wind turbine ranges beyond the 5-km mark, where the effects of terrain occultation are increasingly larger, turbine truncation can be adequately neglected. This situation is easily explained by the fact that, in general, at larger ranges, the turbine Doppler cross section is just a small fraction of the radar-beam illuminated area. Now, the angular radar antenna discrimination is not large enough to be affected by distant wind turbines.

In the horizontal plane, taking into account the rotation of the radar, for wind turbine ranges up to $3 \mathrm{~km}$, the values of impacted azimuths angles remain high, potentially blocking measurement on significant geographical areas (Fig. 2, middle plot). For azimuth off-axis beam impeding angles several times the half-power beamwidth, the Doppler signal will undergo obscuration as far as the discrimination of the principal lobe of the antenna is within the direction of the wind turbine. Similar effects can be also noted in the vertical plane (lower plot in Fig. 2), where obscuration losses are shown in terms of elevation offaxis beam-impeding angles. The lack of symmetry in the plot is associated to terrain effects (in the plot, positive off-axis beam elevation angles move the radar beam toward the terrain).

\section{CONCLUSION}

To analyze the effects of wind turbines blockage on coherent weather radar performance in a realistic way, we have considered the use of simulations of beam propagation in three-dimensional media. The technique divides the medium into slabs, each of which introduces a spatially varying contribution to the phase defined by the atmospheric refraction, and the radar wavebeam is then propagated through a uniform medium from screen to screen. When the radar beam encounters terrain and wind turbines, occultation effect builds up by diffraction over many slabs and produces a complex radiation lobe structure. The large number of spurious radiation lobes in the turbine-modified antenna radiation pattern may challenge the accuracy of radar measurements.

From our analysis, it results clear that wind turbines within $3 \mathrm{~km}$ of meteorological radars can prevent the radar's beam from correctly shaping and cause considerable radar estimation errors down range from the turbines. Our study seems to indicate that, even in simple case scenarios, where only one wind turbine is blocking the radar beam, obscuration losses may be in excess of $2 \mathrm{~dB}$.

\section{REFERENCES}

[1] D. W. Burgess, T. Crum, and R. J. Vogt, "Impacts of wind farms on WSR-88D operations," presented at the 24th Int. Conf. Interactive Inf. Process. Syst. Meteorol., Oceanography, Hydrology, New Orleans, LA, Paper 6B.3.

[2] B. M. Kent1 and K. C. Hill, "Dynamic radar cross section and radar Doppler measurements of commercial general electric windmill power turbines part 1: Predicted and measured radar signatures," IEEE Antennas Propag. Mag., vol. 50, no. 2, pp. 211-219, Apr. 2008.

[3] V. N. Bringi and V. Chandrasekar, Polarimetric Doppler Weather Radar. Cambridge, U.K.: Cambridge Univ.y Press, 2001.

[4] B. R. Mahafza, Radar Systems Analysis and Design. London, U.K.: Chapman \& Hall, 2000.

[5] R. H. Hardin and F. D. Tappert, "Applications of the split-step Fourier method to the numerical solution of nonlinear and variable coefficient wave equations," SIAM Rev., vol. 15, p. 423, 1973.

[6] J. A. Fleck, J. R. Morris, and M. D. Feit, "Time-dependent propagation of high energy laser beams through the atmosphere," Appl. Phys., vol. 10, pp. 129-160, 1976.

[7] J. R. Kuttler and G. D. Dockery, "Theoretical description of the parabolic approximation/Fourier split-step method of representing electromagnetic propagation in the troposphere," Radio Sci., vol. 26, pp. 381-393, 1991.

[8] A. Belmonte, "Feasibility study for the simulation of beam propagation: Consideration of coherent LIDAR performance," Appl. Opt., vol. 39, pp. 5426-5445, 2000. 